Artículo

Los textos publicados son responsabilidad exclusiva de sus autores

\title{
Educación virtual, el disfraz de la enseñanza remota de emergencia
}

\section{Virtual Education, and the remote emergency teaching disguise}

\author{
Diana María Alonzo Mayén \\ Maestría en Docencia Universitaria \\ Universidad de San Carlos de Guatemala \\ https://orcid.org/0000-0003-2613-7331 \\ alonzo.mayen@gmail.com
}

\section{Referencia}

Alonzo Mayen, D. M. (2021). Educación Virtual, el disfraz de la enseñanza remota de emergencia.

Revista Guatemalteca de Educación Superior, 4(2), 11-22.

DOI: https://doi.org/10.46954/revistages.v4i2.58

Recibido: 15/02/2021

Aceptado: 04/04/2021

\section{Resumen}

La pandemia de la COVID-19 generó que de forma abrupta la comunidad educativa del mundo entero implementara medidas para no detener el año escolar. La efectividad del proceso de aprendizaje y la necesidad de una rápida adaptación pueden disminuir la calidad de los recursos y la educación. La Universidad de San Carlos de Guatemala no fue la excepción, he implementó acciones rápidamente. El objetivo principal de este estudio fue analizar las acciones implementadas, esto permitirá desarrollar una estrategia coherente y bien definida de un plan de emergencia para activar en cualquier momento la Enseñanza Remota. Para ello, se aplicó un método de investigación mixto; este se realizó en el Centro Universitario de Suroccidente, tomando como muestra, a los estudiantes y profesores del cuarto semestre 
de la Licenciatura de Ciencias de la Comunicación, durante el mes de marzo hasta noviembre de 2020. Los resultados fueron contrastados con el testimonio brindado por representantes de la División de Educación a Distancia en Entornos Virtuales y el coordinador de la carrera mencionada. La investigación dejó al descubierto muchas debilidades y carencias que tienen no solo los centros regionales sino el equipo docente y los mismos estudiantes. Por ejemplo, la mayoría de estudiantes únicamente tienen teléfono celular para acceder a sus clases, limitándose

Palabras claves:

enseñanza, remota, pandemia, COVID, educación, emergencia, virtual.

\section{Keywords:}

Education, Remote, pandemic, COVID19, teaching, emergency, virtual. así a realizar las actividades colaborativas. Se evidenció que los alumnos están familiarizados a las redes sociales, más no con herramientas educativas aplicadas a la educación.

\section{Abstract}

The COVID-19 pandemic caused the entire world's educational community to abruptly implement measures that would not stop the school year. The effectiveness of the learning process and the need for a rapid adaptation can decrease the quality of resources and education. The University of San Carlos of Guatemala was no exception, it implemented actions quickly. The main objective of this study was to analyze the implemented actions, this will allow to develop a coherent and well-defined strategy of an emergency plan to activate Remote Teaching at any time. To this end, a mixed research method was applied; this was held at the University Center of Suroccidente, taking as a sample, the students and professors of the fourth semester of the Bachelor of Sciences in Communication, from March to November 2020. The results were contrasted with the testimony provided by representatives of the Division of Distance Education in Virtual Environments and the coordinator of the aforementioned career. The research exposed many weaknesses and deficiencies that not only the regional centers have but, the teaching team and the students themselves. For example, the mayority of students only have a cell phone to access their classes, thus limiting themselves to collaborative activities. It was shown that students are familiarized with social media, but not with educational tools applied to education. 


\section{Introducción}

La pandemia de la COVID-19 ha tenido un fuerte impacto en el sector educativo, en más de 180 países del mundo que se han visto obligados a tomar medidas drásticas, suspendiendo actividades presenciales y optando por sesiones a distancia a través de la virtualidad para no detener el aprendizaje. Esta situación de emergencia en la salud, donde el distanciamiento social es necesario, el confinamiento obligatorio y la reducción de conglomerados es decisiva para evitar la propagación del virus, ha incidido notablemente en la educación en todos los nivel, obligando a los docentes y estudiantes a replantearse su manera de orientar la actividad pedagógica y así como la forma de aprendizaje y haciendo del internet la herramienta más idónea para continuar con el desarrollo de las actividades escolares, tal como lo plantean Mancera, Serna y Barrios (2020), quienes sostienen que frente a la contingencia por la pandemia:

...maestras y maestros han respondido con los medios que están a su alcance para mantener el quehacer educativo. Si WhatsApp o correo electrónico son los recursos más ampliamente disponibles y que más fácilmente permiten llegar a las familias, son estos en los que los docentes se apoyan (s/p).

La disposición de reorganizar las actividades académicas a distancia y haciendo uso de las TIC fue girada para los diferentes niveles educativos, desde la educación preescolar hasta las universidades; con ello, en cuestión de días, docentes y alumnos quedaron anclados a un entorno digital improvisado al que muchos han tenido que acostumbrarse de forma repentina, y a veces hasta sin contar con los recursos adecuados para la conectividad.

Ante esta nueva modalidad, muchas personas han confundido el término y suelen hacer comparaciones erróneas al referirse a este plan de emergencia como la nueva modalidad educativa. Es imperante repetir que estas son medidas paliativas para no detener el año escolar y esto no determina la efectividad del 
proceso de aprendizaje, ya que con la necesidad de una rápida adaptación se puede disminuir la calidad de los recursos y la educación en general, pero lo importante, era continuar con el proceso educativo.

En este sentido, es propicio señalar lo expuesto por Torres (2020) quien sostiene que:

El objetivo principal en estas circunstancias no es recrear un ecosistema educativo robusto, sino más bien proporcionar acceso temporal a los materiales de enseñanza de una manera rápida y fácil de configurar. Cuando se entienden las ERT de esta manera, es más fácil diferenciarlas del concepto "aprendizaje online".

Los investigadores en tecnología educativa, específicamente en la subdisciplina del aprendizaje en línea y a distancia, han definido cuidadosamente términos a lo largo de los años para distinguir entre las soluciones de diseño altamente variables que se han desarrollado e implementado: aprendizaje a distancia, aprendizaje distribuido, aprendizaje mixto, aprendizaje en línea, dispositivos móviles. Aprendizaje y otros.

Sin embargo, la comprensión de las importantes diferencias no se ha extendido más allá del mundo aislado de los investigadores y profesionales de la tecnología educativa y el diseño instruccional.

Es por ello que en el artículo científico publicado en la revista Educase por (Charles Hodges, 2020) abre el análisis y propone un nuevo concepto "queremos ofrecer una discusión importante en torno a la terminología y proponer formalmente un término específico para el tipo de instrucción que se imparte en estas circunstancias apremiantes: enseñanza remota de emergencia".

Con esta modalidad remota se ha llevado a cabo en los últimos meses, el proceso de enseñanza, y surgen diversas interrogantes, pero principalmente ¿En qué medida la estrategia y el plan de acción fueron suficientes para manejar la enseñanza remota de 
emergencia en los centros regionales? Considerando que mucho de estos, acogen a estudiantes que viven en los sectores más vulnerables, pobres y recónditos del país.

Por tal razón, debemos hacer una lista de lecciones aprendidas, ya que al observar la experiencia de las estrategias implementadas de emergencia pueden ser útiles como punto de partida para plantearnos los modos, métodos, y canales de entrega que debe llevar un modelo pedagógico en un futuro próximo.

Pero más visionario aún, es hacer hincapié en la necesidad que representa tener el diseño y estructura bien definida de un plan de emergencia para echar andar en cualquier momento la Enseñanza Remota de Emergencia; considerando los factores externos que empañan muchas veces el proceso educativo en la Universidad de San Carlos de Guatemala, tales como: huelgas, manifestaciones, situaciones climáticas, tomas de edificios y muchos otros más.

Con lo anteriormente expuesto se definieron los objetivos de la investigación, para la evaluación de variables concretas que permitieran: Analizar la estrategia y el plan de emergencia para llevar a cabo la Educación Remota de Emergencia en el Centro Universitario de Suroccidente.

\section{Materiales y métodos}

Se utilizó un enfoque mixto, con alcance descriptivo, diseño concurrente. En este sentido, se tomaron los cuatro objetivos planteados:Objetivouno:Identificarla infraestructuratecnológica. Objetivo dos: Establecer competencias tecnológicas. Objetivo tres: Determina la orientación proporcionada. Objetivo cuatro: Enlistar dificultades o limitantes presentadas.

A través de las técnicas: grupos focales, entrevistas abiertas a: coordinador de la carrera, un docente técnico de la DEDEV y, la encuesta a 13 estudiantes de la carrera, se realizó la recopilación de información. 
Para realizar el estudio se tomó una muestra a conveniencia y de personas voluntarias. Conformada de docentes, cursos y estudiantes de la Licenciatura en Ciencias de la Comunicación del Centro Universitario del Suroccidente.

Se realizó un análisis descriptivo y de varianza, contenidos, infraestructura tecnológica y guías docentes. La selección de la muestra fue voluntaria (Otzen \& Manterola, 2017), ya que se recolectaron los datos en unidades académicas que autorizaron la recolección y que los estudiantes aceptaron de forma voluntaria y sin pago alguno participar. "La elección de los participantes depende de circunstancias muy variadas. A esta clase de muestra también se le puede llamar autoseleccionada, ya que las personas se proponen como participantes en el estudio o responden a una invitación" (Hernández Sampiei, R., Fernández Collado, C., \& Bautista Lucio, P.)

El tipo de muestra fue la "Homogénea"; ya que participaron todos los estudiantes del cuarto semestre del nivel técnico, esto permitió la participación y la recolección de datos sin distinción alguna. "En las muestras homogéneas las unidades que se van a seleccionar poseen un mismo perfil o características, o bien comparten rasgos similares. Su propósito es centrarse en el tema por investigar o resaltar situaciones, procesos o episodios en un grupo social." (Hernández Sampiei, R., Fernández Collado, C., \& Bautista Lucio, P.)

Para el análisis de resultados se realizó una triangulación de la información, porque esto permitió contrastar la información que aportaron los estudiantes con la que brindaron los docentes y directivos.

\section{Resultados}

Para efecto de esta publicación, nos concentraremos en las evidencias obtenidas, que refiere a enlistar las dificultades o limitantes presentadas en el desarrollo del plan de Enseñanza Remota de Emergencia. Lo evidenciado en el grupo focal y las entrevistas fueron contrastados con los datos cuantitativos que arrojó la encuesta girada a estudiantes. 
TABLA DE ANÁLISIS OBJETIVO 4

\begin{tabular}{|l|r|r|}
\hline Variables & Segmentos & Porcentajes \\
\hline Interfaz del usuario limitada & 5 & 71.43 \\
\hline $\begin{array}{l}\text { Limitación de internet en } \\
\text { zonas rurales (+) }\end{array}$ & 2 & 28.57 \\
\hline $\begin{array}{l}\text { La plataforma no es } \\
\text { adecuada para el celular }\end{array}$ & 2 & 28.57 \\
\hline $\begin{array}{l}\text { Afecta cognitiva y } \\
\text { emocionalmente }\end{array}$ & 1 & 28.57 \\
\hline $\begin{array}{l}\text { Profesores carentes de } \\
\text { alfabetización digital }\end{array}$ & 1 & 14.29 \\
\hline Presupuesto escaso & 1 & 14.29 \\
\hline $\begin{array}{l}\text { Equipo de cómputo } \\
\text { adecuado }\end{array}$ & 14 & 14.28 \\
\hline Total & & 100 \\
\hline
\end{tabular}

Fuente: Investigación 2021

Como se ve en la tabla de datos la extracción del segmento: interfaz limitada es la variable más contante lo que implica que en global de las opiniones sugeridas por el grupo focal y las entrevistas particulares un 28,57\% de la opinión establecida señalan que "la mayoría de estudiantes ingresa por un teléfono celular y utiliza tarjetas prepago, además la plataforma no permite muchas veces interactuar, o el teléfono es muy viejo y no la aguanta".

En este orden de ideas el gráfico de análisis queda de la siguiente manera: el $28,57 \%$ cree que los estudiantes y profesores se puede conectar con sus celulares pero su capacidad de memoria debido a lo desactualizado de sus procesadores, programas y licencias no hacen posible descargar los programas adecuados, por lo que el un 14,29\% opina que la mejor manera de conectarse es por medio de computadoras, pero que no hay presupuesto para ello y en consecuencia es necesario continuar trabajando con las herramientas que se tengan a mano. 
Otro segmento que avala los indicadores propuestos es que los docentes entrevistados coincidieron en que la debilidad de la ERT, está en que muchos estudiantes y profesores viven en áreas rurales muy remotas, donde la señal del Internet es muy baja. Finalmente, el $28.57 \%$ señalan en sus entrevistas que este hecho ha afectado emocionalmente a los estudiantes

Para los datos cuantitativos, la encuesta fue aplicada a 13 alumnos. La muestra señaló compatibilidad con los datos suministrados por los profesores. A continuación, el detalle de lo consolidado referente al objetivo para identificar limitaciones y dificultades.

Tabla No. 1. ¿Con qué dispositivo se conecta a su clase?

\begin{tabular}{|l|r|l|}
\hline Variable & Segmento & Porcentaje \\
\hline Celular & 4 & $31 \%$ \\
\hline Tablet & 2 & $15 \%$ \\
\hline Computadora & 7 & $54 \%$ \\
\hline Total & 13 & $100 \%$ \\
\hline
\end{tabular}

Fuente: Investigación 2021

Tabla No. 2. ¿Qué dificultades ha tenido para poder conectarse a sus clases virtuales?

\begin{tabular}{|l|r|r|}
\hline Variable & Segmento & Porcentaje \\
\hline Mala señal de internet & 3 & $23 \%$ \\
\hline No tengo acceso a internet & 2 & $15 \%$ \\
\hline $\begin{array}{l}\text { Mi dispositivo no es compatible } \\
\text { con las opciones de la } \\
\text { plataforma }\end{array}$ & 2 & $15 \%$ \\
\hline $\begin{array}{l}\text { Se pierde señal por fenómenos } \\
\text { atmosféricos }\end{array}$ & & \\
\hline Total & 13 & $100 \%$ \\
\hline
\end{tabular}

Fuente: propia, investigación 2021. 


\section{Discusión}

¿Educación virtual? No nos confundamos, y es necesario entender que lo que se ha aplicado en el proceso educativo a lo largo de la pandemia, es Enseñanza Remota y no Educación virtual; teniendo enfatizado esto, podemos continuar con la discusión de lo evidenciado en la investigación. Por lo tanto, no se pretende entonces hacer un análisis conclusivo, sino de enfocar y comprobar la propuesta de hipótesis de comprobar que la Enseñanza Remota de Emergencia (ERT) es una alternativa de enseñanza plausible que se vinculará con las tecnologías y que debe establecerse un plan bien definido para implementarse en cualquier momento si así lo amerita la situación.

Cuando se planteó la idea de evaluar la Educación Remota Emergencia, varios expertos apuntaban que lo más aconsejable era analizar el proceso llevado y no evaluado porque no se tenía un punto de comparación, como ya lo mencionamos, no podemos comparar la educación virtual con la educación remota, porque la primera fue diseñada en su génesis así y lleva una metodología clara, coherente, ordenada y que permite evaluar y medir los objetivos alcanzados.

En el artículo especializado "La diferencia entre la enseñanza remota de emergencia y el aprendizaje en línea" los académicos refieren que, si bien no existe un modelo para evaluar la ERT, puede tomarse como base el lenguaje del modelo CIPP: "la evaluación de ERT requerirá preguntas más amplias, especialmente durante las implementaciones iniciales, dónde debe enfocar su evaluación relacionada con los esfuerzos de ERT. Se utilizará el lenguaje del modelo CIPP para la estructura. CIPP es un acrónimo que representa contexto, insumos, proceso y productos" (Charles Hodges, 2020).

La investigación claramente refleja que la Universidad de San Carlos de Guatemala, a pesar de los pequeños esfuerzos realizados no cuenta con la infraestructura tecnológica adecuado para dar un soporte de tal magnitud; mucho de esto tiene que ver con la asignación presupuestaria para la compra de servidores y equipo tecnológico y por ende para la contratación del recurso humano técnicamente capacitado para tal fin. 
En cuanto a las limitaciones y dificultades se evidencian que la interfaz del usuario es limitada, la plataforma no es la adecuada, el impacto emocional que afectó a la comunidad educativa, limitación de los servicios de internet en zonas rurales, presupuesto escaso, carencia de equipo de cómputo y profesores carentes de alfabetización digital.

Se logró comprobar que los docentes y alumnos no contaban con la experiencia para el uso óptimo de herramientas y aplicaciones, se determinó que la mayoría de estudiantes no cuentan con equipo de cómputo para recibir sus clases y lo hacen a través del teléfono celular.

Sin embargo, la mayor dificultad es que la plataforma no es muy amigable y la mayoría de los estudiantes se conectan a través del teléfono celular, a esto también es relevante agregar los fenómenos climatológicos como intensa lluvia y la infraestructura de las viviendas de los estudiantes y docentes.

En conclusión, esta investigación no pretende ser crítica sino al contrario ser visionaria y que sirva de antecedente para trazar los lineamentos de un plan de Enseñanza Remota de Emergencia y dejar claro que un modelo pedagógico diseñado para impartirse de forma presencial no puede modificarse a lo virtual, sino que debe diseñar desde su inicio con esa intención.

\section{Agradecimientos}

Sencillo no ha sido el proceso, pero gracias al Centro Universitario de Suroccidente por la apertura para realizar la investigación. A la División de Educación a Distancia en Entornos Virtuales, en especial al Dr. Carlos Chiquitó, a mis docentes por el acompañamiento, en especial a la Dra. Walda Flores por su constante motivación. A la Dra. Alice Burgos por esas palabras alentadoras y por inspirarme. 


\section{Referencias}

Charles Hodges, S. M. (27 de Marzo de 2020). La diferencia entre la enseñanza remota de emergencia y el aprendizaje en línea. Obtenido de EDUCAUSE REVIEW: https://er.educause.edu/articles/2020/3/the-differencebetween-emergency-remote-teaching-and-onlinelearning

Mancera, S. y. (2020). Pandemia: maestros, tecnología y desigualdad. Nexo. Obtenido de https://educacion. nexos.com. $\mathrm{mx} / \mathrm{p} \mathrm{p}=2286$

Otzen Tamara, M. C. (2017). Técnicas de Muestreo sobre una población a estudio. Int. J. Morphol. https://doi. org/10.4067/S0717-95022017000100037

Sampieri, R. H. (2018). Metodología de la Investigación. México, D.F: McGrawHill.

Torres, D. (2020). Obtenido de https://www.torresburriel.com/ weblog/2020/04/10/en-que-consiste-la-ensenanzaremota-en-emergencias-ere/ 


\section{Sobre la autora}

\section{Diana María Alonzo Mayén}

Es Licenciada en Ciencias de la Comunicación Social. Presentadora del noticiero "Telediario" y TN23. Voz oficial para EEUU y Latinoamérica, del Canal Internacional "Telecentro Internacional" de la Corporación Alba Visión. Asesora en estrategias de comunicación institucional, y en manejo de información sensible en el tema de drogas y prevención de la violencia.

Usted es libre para compartir, copiar y redistribuir el material en cualquier medio o formato y adaptar el documento, remezclar, transformar y crear a partir del material para cualquier propósito, incluso comercialmente, siempre que cumpla la condición de atribución: usted debe reconocer el crédito de una obra de manera adecuada, proporcionar un enlace a la licencia, e indicar si se han realizado cambios. Puede hacerlo en cualquier forma razonable, pero no de forma tal que sugiera que tiene el apoyo del licenciante o lo recibe por el uso que hace. 